\title{
Banco de sementes de espécies arbóreas em floresta estacional no Rio Grande do Sul, Brasil ${ }^{1}$
}

\author{
CAROLINE SCHERER ${ }^{2}$ e JOÃO ANDRÉ JARENKOW ${ }^{2,3}$
}

(recebido: 5 de agosto de 2004; aceito: 5 de janeiro de 2006)

\begin{abstract}
Arboreal seed bank in a seasonal forest in Rio Grande do Sul, Brazil). Soil seed bank composition provides basic informations about the potential regeneration of a community, allowing further inferences about successional processes. In a permanent 0.5 ha plot (50 square $100 \mathrm{~m}^{2}$ sample units) of seasonal forest located on a south facing slope in the State Park of Itapuã, municipality of Viamão (RS), the composition and distribution of tree species in the soil seed bank were analyzed. Litter and soil from two depths $(0-5$ and $5-10 \mathrm{~cm})$ were collected in all sample units, in the spring (September 2002) and fall (March 2003). The collected material was left to germinate in a greenhouse under two distinct light conditions, natural and with a lattice-shade cover $(50 \%)$. Species composition and density was relatively similar (Jaccard index $=0.67$ ) for the two seasons. A comparison with the known tree composition of the same area indicated a very low similarity, for both seasons. The absence of significative difference between periods may reflect a relatively homogeneous humid subtropical condition, in which the seasonality does not show strength. The presence of both shade-tolerant and light-demanding species in the seed bank indicates a high potential of regeneration for the tree component, in the case of gap formation or other disturbances to the present forest structure.
\end{abstract}

Key words - forest regeneration, seasonal forest, soil seed bank, tree component

RESUMO - (Banco de sementes de espécies arbóreas em floresta estacional no Rio Grande do Sul, Brasil). O conhecimento da composição do banco de sementes do solo fornece informações básicas sobre o potencial de regeneração da comunidade, permitindo que se façam inferências sobre o processo sucessional. Em uma parcela permanente de 0,5 ha (50 unidades amostrais de $100 \mathrm{~m}^{2}$ ) localizada em floresta estacional de encosta com exposição sul no Parque Estadual de Itapuã, município de Viamão (RS), foram analisadas a composição e a distribuição de espécies arbóreas no banco de sementes do solo. Em todas as unidades amostrais foram coletadas a serapilheira e o solo, este em duas profundidades $(0-5 \mathrm{~cm} \mathrm{e} 5-10 \mathrm{~cm})$, na primavera (setembro 2002) e no outono (março 2003). O material coletado foi colocado a germinar em casa de vegetação, sob duas distintas condições de luz: natural e com recobrimento de sombrite (50\%). A composição de espécies e a densidade foram relativamente similares para as duas estações (índice de Jaccard $=0,67$ ). Em comparação com a composição arbórea atual da área a similaridade foi muito baixa, para ambas estações. A ausência de diferenças significativas entre os períodos de coleta pode refletir a condição relativamente homogênea subtropical úmida, na qual a estacionalidade não se mostra efetiva. A presença de espécies dependentes de luz e de tolerantes à sombra no banco de sementes, indica um alto potencial de regeneração para o componente arbóreo, no caso de formação de clareira ou outras perturbações na estrutura florestal presente.

Palavras-chave - banco de sementes do solo, componente arbóreo, mata estacional, regeneração florestal

\section{Introdução}

O banco de sementes do solo é uma das principais fontes de recrutamento de novos indivíduos em fases iniciais da sucessão (Hall \& Swaine 1980, Butler \& Chazdon 1998). Todas as sementes viáveis presentes no solo constituem o banco de sementes (Thompson \& Grime 1979, Garwood 1989), o qual é continuamente

1. Parte da dissertação de mestrado do primeiro autor, Programa de Pós-Graduação em Botânica da Universidade Federal do Rio Grande do Sul.

2. Universidade Federal do Rio Grande do Sul, Departamento de Botânica, Av. Bento Gonçalves, 9500, 91501-970 Porto Alegre, RS

3. Autor para correspondência: jarenkow@portoweb.com.br enriquecido pela chuva de sementes (Harper 1977). Nos ecossistemas florestais, os propágulos que alcançam o solo são produzidos predominantemente por espécies encontradas no local, pela liberação direta de sementes de frutos, e por outras de áreas vizinhas e mesmo de locais mais distantes, dada a eficiência de seus mecanismos de dispersão (Martínez-Ramos \& SotoCastro 1993). A contribuição destas diferentes fontes de propágulos determina a estrutura da comunidade florestal, sendo essencial proteger os remanescentes de florestas para manter estas fontes (Espíndola et al. 2003).

O banco de sementes do solo é um produto da história do local (Fenner 1985), uma vez que espécies não presentes podem nele persistir até o surgimento de 
condições favoráveis para a sua germinação e estabelecimento (Williams-Linera 1993). O período em que a semente permanece viável no solo depende de seus atributos fisiológicos (tipo de dormência), de interações bióticas (existência de parasitas e/ou predadores) e de condições abióticas (disponibilidade de água, luz e oxigênio) (Garwood 1989).

Alterações temporais na composição florística de uma comunidade, variações sazonais na frutificação e na dispersão influenciam na abundância de sementes, no número de espécies e nas formas de vida disponíveis no solo de uma comunidade durante o ano ou de ano para ano (Young et al. 1987, Alvarez-Buylla \& MartínezRamos 1990, Dalling et al. 1997, 1998). Existem evidências de que perturbações e fragmentação também podem influenciar na riqueza e na abundância de espécies no solo (Young et al. 1987, Graham \& Hopkins 1990, Baider et al. 2001).

Atualmente as pesquisas buscam a compreensão da organização dos ecossistemas a fim de obterem-se métodos racionais para a resolução dos problemas ambientais. O entendimento dos processos de regeneração natural da vegetação, após um distúrbio, é fundamental para delinear os procedimentos mais adequados à restauração e manutenção da diversidade (Gross 1990).

No Brasil, recentemente alguns estudos têm se preocupado em quantificar o banco de sementes do solo em florestas, destacando-se os trabalhos de Daniel \& Jankauskis (1989), Roizman (1993), Baider et al. (1999, 2001) e Grombone-Guaratini \& Rodrigues (2002). No sul do Brasil, até o momento, há apenas o trabalho de Caldato et al. (1996), realizado em dois tipos florestais: com predominância da araucária e com predominância de outras espécies nativas.

Este estudo teve como objetivos obter a composição e a densidade de sementes de espécies arbóreas presentes no banco de sementes do solo e avaliar as possíveis variações no banco entre as profundidades, entre as épocas distintas de coleta de solo e as diferenças sazonais na composição das amostragens, como também verificar a similaridade do banco com a vegetação já estabelecida na área.

\section{Material e métodos}

O estudo foi desenvolvido no Parque Estadual de Itapuã, no Município de Viamão, Rio Grande do Sul, localizado entre as coordenadas $30^{\circ} 20^{\prime}$ a $30^{\circ} 27^{\prime} \mathrm{S}$ e $50^{\circ} 55^{\prime}$ a $51^{\circ} 05^{\prime} \mathrm{W}$. O Parque tem uma área de 5.566 ha, distribuída entre sistemas aquáticos e terrestres, com altitudes que variam de cinco a 263 m.s.m.
O clima na região da Grande Porto Alegre, na qual se localiza a área de estudo, é subtropical úmido, do tipo Cfa, na classificação de Köppen, com temperatura média anual de $17,5^{\circ} \mathrm{C}$, e médias do mês mais quente e mais frio de $24,6^{\circ} \mathrm{C}$ e $13,8{ }^{\circ} \mathrm{C}$, respectivamente (Rio Grande do Sul 1997). A precipitação média anual é de $1.322 \mathrm{~mm}$, com as chuvas bem distribuídas durante o ano (Moreno 1961). Na área do Parque coexistem as formações do Escudo Cristalino Sul-RioGrandense, que compreende a faixa granítico-magmatítica (Soliani et al. 2000), representada por coxilhas e morros, e a da Planície Costeira, que apresenta relevos com extensas superfícies planas ou levemente onduladas, que no extremo leste se associam aos grandes banhados em fase de colmatação e avançam até o limite das praias arenosas lacustres (Rio Grande do Sul 1997). Os principais grupos de solos que ocorrem no Parque são oriundos da intemperização do granito e derivados de sedimentos (Rio Grande do Sul 1997). Conforme Kray (2004), o solo é classificado como de textura média, apresentando teores médios de matéria orgânica, e com pH muito baixo, não apresentando caráter álico. A baixa saturação de bases mostra que se trata de um solo distrófico.

A cobertura vegetal na região em que se insere o Parque, segundo Veloso \& Góes-Filho (1982), é condicionada por fatores ecológicos especiais que definem uma "área de tensão ecológica". Estes fatores, determinados pelo encontro de duas regiões fitoecológicas distintas, neste caso a savana e a floresta estacional, levam a uma flora que consiste da interpenetração de suas vegetações.

O remanescente florestal no qual se realizou o estudo recobre toda a encosta sul do morro do Campista, em frente à praia do Tigre, desde a linha da praia até o topo do morro e, lateralmente, estende-se pelas encostas adjacentes, caracterizado como floresta estacional (Kray 2004). A área apresenta-se em bom estado de preservação e com poucos afloramentos rochosos, sendo denominada de zona primitiva, por apresentar pouca intervenção humana no passado (Rio Grande do Sul 1997). Na parcela estudada inexistem clareiras e o componente herbáceo é esparso, com sub-bosque pouco denso e algumas lianas. O componente arbóreo é de porte pouco elevado (alturas até $16 \mathrm{~m}$ ) devido aos afloramentos rochosos e solos rasos, no qual destacam-se como estruturalmente importantes Guapira opposita (Vell) Reitz, Trichilia claussenii C. DC., Eugenia rostrifolia D. Legrand, Garcinia gardneriana (Planch. \& Triana) Zappi, Roupala brasiliensis Klotzsch, entre outras (Kray 2004).

A amostragem do banco de sementes do solo (BSS) foi feita em uma parcela permanente de 0,5 ha, constituída de 50 unidades amostrais de $10 \mathrm{~m}$ de lado $\left(100 \mathrm{~m}^{2}\right)$, com amostragem em duas épocas distintas: na primavera (setembro 2002 BSS 1) e no outono (março 2003 - BSS 2). Em cada unidade amostral foram coletados dois pontos: o primeiro foi no centro da unidade amostral, ou seja, no ângulo de um quadrado de $5 \mathrm{~m}$ de lado e, o segundo, foi no ângulo de um quadrado de $2 \mathrm{~m}$ de lado, todos com o ponto de origem comum. Para a 
segunda amostragem (outono), os pontos de coleta foram feitos o mais próximo do ponto da amostragem anterior.

Em cada ponto coletou-se a serapilheira e duas amostras de solo em profundidades distintas (entre a superfície e $5 \mathrm{~cm}$ e outra entre $5 \mathrm{~cm}$ e $10 \mathrm{~cm}$ de profundidade), com um amostrador de metal ( $20 \mathrm{~cm}$ de diâmetro e $5 \mathrm{~cm}$ de altura). A área total de solo coletado em cada época foi de $1,97 \mathrm{~m}^{2}$. A serapilheira e as amostras tomadas em cada unidade amostral e na mesma profundidade foram misturadas e acondicionadas em sacos plásticos, rotulados e transportados para o local da instalação do experimento, constituindo 50 amostras compostas para cada nível.

Após a homogeneização, uma camada de cerca de $2 \mathrm{~cm}$ de cada amostra composta foi disposta em duas bandejas de $27 \mathrm{~cm}$ de diâmetro, sobre uma base de vermiculita, e colocadas a germinar em tratamentos distintos: sob incidência de luz natural (luz) e com recobrimento de sombrite (50\%) (sombra). Cada tratamento constou de 150 bandejas (50 de cada nível), mais quatro bandejas contendo areia esterilizada, como controle de contaminação. As bandejas foram regadas quando necessário, de modo a manter as condições de umidade adequadas à germinação.

O método utilizado para a quantificação das plântulas de espécies arbóreas foi o de emergência de plântulas ou germinação (Gross 1990, Brown 1992). A germinação foi acompanhada semanalmente durante 20 semanas, retirando-se da bandeja os indivíduos de espécies conhecidas, tendo cuidado para não remover o solo. Os indivíduos jovens, cuja identificação permaneceu duvidosa, foram transplantados para recipientes maiores, até desenvolverem-se a ponto de possibilitar o seu reconhecimento. Ao final das 20 semanas, o solo foi revolvido para promover a germinação de sementes que eventualmente tivessem ficado sem condições de desenvolvimento, acompanhando-se a germinação por mais quatro semanas. Os indivíduos germinados na serapilheira foram somados aos germinados na profundidade entre a superfície e $5 \mathrm{~cm}$. O experimento foi instalado na casa de vegetação do Departamento de Solos, da Faculdade de Agronomia, da Universidade Federal do Rio Grande do Sul.

A identificação das espécies foi feita por consulta à bibliografia específica, por comparação com o material de herbário ou com auxílio de especialista. A delimitação de famílias seguiu as sugestões de APG II (2003), nas quais foram incluídas as espécies determinadas.

Os parâmetros fitossociológicos estimados foram os de densidade relativa, frequiência absoluta e relativa e o valor de importância (Mueller-Dombois \& Ellenberg 1974), assim como a diversidade específica pelo índice de Shannon $\left(H^{\prime}\right)$, e a equabilidade pelo índice de Pielou $\left(J^{\prime}\right)$ (Magurran 1988). Foram calculadas as similaridades florísticas, pelo índice de Jaccard (Mueller-Dombois \& Ellenberg 1974), entre BSS 1 e BSS 2, e entre estes e o componente arbóreo estabelecido na área de estudo, a partir de levantamento realizado na área por Kray (2004), que incluiu indivíduos com diâmetro à altura do peito (DAP) igual ou superior a $5 \mathrm{~cm}$.
As relações entre os tratamentos e as profundidades do solo foram verificadas através da análise do número médio de espécies e do número médio de sementes em cada amostra. Foi utilizado o teste de Kolmogorov-Smirnov para verificação da normalidade dos dados e da homogeneidade entre variâncias. Como estes não apresentaram normalidade e/ou não-homogeneidade entre variâncias, aplicou-se a análise de variância não-paramétrica para um fator ou teste de Kruskal-Wallis (Callegari-Jacques 2004). Posteriormente, as médias foram comparadas aos pares pelo teste de Tukey, através de um procedimento de comparação múltipla nãoparamétrica (Zar 1999).

\section{Resultados}

A riqueza no banco de sementes do solo na amostragem de primavera (BSS 1), para ambos tratamentos, foi de 19 espécies arbóreas, distribuídas em 16 gêneros e dentro de 13 famílias e um indivíduo identificado até ao nível de família (tabela 1). $\mathrm{Na}$ amostragem de outono (BSS 2), em ambos os tratamentos, germinaram 16 espécies arbóreas, de 13 gêneros e 11 famílias (tabela 1). As famílias que apresentaram a maior riqueza foram Solanaceae e Salicaceae, com três espécies cada, seguidas de Moraceae, Sapindaceae e Urticaceae, com duas, e as demais com somente uma espécie (tabela 1).

No BSS 1 germinaram 895 indivíduos, resultando em uma densidade de sementes viáveis no solo de 78,17 sementes $\mathrm{m}^{-2}$, enquanto no BSS 2 germinaram 807 indivíduos com uma densidade de 74,63 sementes $\mathrm{m}^{-2}$.

As espécies com as maiores densidades no BSS 1 e no BSS 2 foram Banara parviflora, Ficus organensis e Trema micrantha. Estas três espécies acumularam $85 \%$ e $82 \%$ do total de indivíduos germinados e apresentam-se também como as primeiras em valor de importância que juntas acumularam 79\% e $74 \%$ do total, respectivamente (tabelas 2, 3).

No BSS 1 as sete espécies que apresentaram maior número de indivíduos germinados representaram $96 \%$ do total, e destas, seis foram comuns a todos os tratamentos: Ficus organensis, Trema micrantha, Banara parviflora, Ficus luschnathiana, Solanum mauritianum e Cereus hildmannianus, exceto Casearia silvestris, que germinou apenas no tratamento recoberto com sombrite. As outras 13 espécies acumularam os $4 \%$ restantes (tabela 4 ).

$\mathrm{Na}$ primeira amostragem, no tratamento com sombrite, germinaram 649 indivíduos ( $72 \%$ do total do BSS 1), estando representadas todas as espécies recrutadas na totalidade deste experimento (figura 1A). Quanto à profundidade, os maiores valores ocorreram 
Tabela 1. Espécies e número de indivíduos recrutados no banco de sementes do solo (BSS 1; BSS 2) em uma floresta estacional no Parque Estadual de Itapuã, Viamão, RS, nas suas respectivas famílias.

Table 1. Species and individuals recruited from soil seed bank (BSS 1; BSS 2) in a seasonal forest at State Park of Itapuã, Municipality of Viamão (RS), with the respective family.

\begin{tabular}{|c|c|c|}
\hline \multirow[t]{2}{*}{ Família/Espécie } & \multicolumn{2}{|c|}{ Número de indivíduos } \\
\hline & BSS 1 & BSS 2 \\
\hline \multicolumn{3}{|l|}{ ARECACEAE } \\
\hline Syagrus romanzoffiana (Cham.) Glassman & 2 & - \\
\hline \multicolumn{3}{|l|}{ BORAGINACEAE } \\
\hline Patagonula americana $\mathrm{L}$. & 2 & 1 \\
\hline \multicolumn{3}{|l|}{ CACTACEAE } \\
\hline Cereus hildmannianus K. Schum. & 14 & 16 \\
\hline \multicolumn{3}{|l|}{ CANNABACEAE } \\
\hline Trema micrantha $(\mathrm{L}$.$) Blume$ & 259 & 162 \\
\hline \multicolumn{3}{|l|}{ CLUSIACEAE } \\
\hline Garcinia gardneriana (Planch. \& Triana) Zappi & 1 & 2 \\
\hline \multicolumn{3}{|l|}{ EUPHORBIACEAE } \\
\hline Sebastiania serrata (Klotzsch) Müll. Arg. & 1 & - \\
\hline \multicolumn{3}{|l|}{ MORACEAE } \\
\hline Ficus luschnathiana (Miq.) Miq. & 41 & 13 \\
\hline Ficus organensis (Miq.) Miq. & 340 & 224 \\
\hline \multicolumn{3}{|l|}{ MYRTACEAE } \\
\hline Eugenia rostrifolia D. Legrand & - & 2 \\
\hline Myrtaceae 1 & 1 & - \\
\hline \multicolumn{3}{|l|}{ ROSACEAE } \\
\hline Prunus myrtifolia (L.) Urb. & 1 & - \\
\hline \multicolumn{3}{|l|}{ RUTACEAE } \\
\hline Zanthoxylum rhoifolium Lam. & 8 & 17 \\
\hline \multicolumn{3}{|l|}{ SALICACEAE } \\
\hline Banara parviflora (A. Gray) Benth. & 163 & 275 \\
\hline Casearia silvestris $\mathrm{Sw}$. & 22 & 54 \\
\hline Salix humboldtiana Willd. & 3 & - \\
\hline \multicolumn{3}{|l|}{ SAPINDACEAE } \\
\hline Allophylus edulis (A. St.-Hil.) Radlk. & 1 & - \\
\hline Cupania vernalis Cambess. & - & 4 \\
\hline \multicolumn{3}{|l|}{ SOLANACEAE } \\
\hline Solanum mauritianum Scopoli & 21 & 12 \\
\hline Solanum pseudoquina A. St.-Hil. & 3 & 1 \\
\hline Solanum sanctaecatharinae Dunal & 2 & 6 \\
\hline \multicolumn{3}{|l|}{ URTICACEAE } \\
\hline Cecropia pachystachya Trec. & 6 & 12 \\
\hline Coussapoa microcarpa (Schott) Rizzini & 4 & 6 \\
\hline Totais & 895 & 807 \\
\hline
\end{tabular}

entre a superfície e $5 \mathrm{~cm}(58 \%)$ e, entre 5 e $10 \mathrm{~cm}$ de profundidade, o restante (42\%) (figura 1A). Na riqueza específica, houve igualmente um decréscimo de 18 para 12 espécies (figura 1B), sendo oito espécies exclusivas na profundidade entre a superfície e $5 \mathrm{~cm}$ (Allophylus edulis, Garcinia gardneriana, Myrtaceae 1, Patagonula americana, Prunus myrtifolia,
Sebastiania serrata, Solanum sanctaecatharinae e Syagrus romanzoffiana) e, duas exclusivas entre 5 e $10 \mathrm{~cm}$ (Salix humboldtiana e Solanum pseudoquina).

No BSS 2, as sete espécies que tiveram o maior número de indivíduos germinados representaram 94\% do total, e destas, cinco foram comuns a todos os tratamentos: Banara parviflora, Ficus organensis, 
Tabela 2. Parâmetros fitossociológicos calculados para as espécies arbóreas recrutadas nas amostras de banco de sementes 1 (BSS 1) em uma floresta estacional no Parque Estadual de Itapuã, Viamão, RS, em ordem decrescente de valor de importância (VI). $(\mathrm{Ni}=$ número de indivíduos; $\mathrm{DR}=$ densidade relativa; $\mathrm{FA}=$ freqüência absoluta; $\mathrm{FR}=$ freqüência relativa).

Table 2. Phytosociological parameters calculated for the tree species recruited in the samples of soil seed bank 1 (BSS 1) in a seasonal forest at State Park of Itapuã, Municipality of Viamão (RS), ranked according to importance value (VI). (Ni = individuals number; DR = relative density; FA = absolute frequency; $F R=$ relative frequency).

\begin{tabular}{|c|c|c|c|c|c|}
\hline Espécie & $\mathrm{Ni}$ & $\begin{array}{l}\mathrm{DR} \\
(\%)\end{array}$ & $\begin{array}{l}\text { FA } \\
(\%)\end{array}$ & $\begin{array}{l}\mathrm{FR} \\
(\%)\end{array}$ & $\begin{array}{c}\mathrm{VI} \\
(\%)\end{array}$ \\
\hline Ficus organensis & 340 & 37,99 & 46,5 & 25,14 & 31,56 \\
\hline Trema micrantha & 259 & 28,94 & 54,0 & 29,19 & 29,06 \\
\hline Banara parviflora & 163 & 18,21 & 35,0 & 18,92 & 18,57 \\
\hline Ficus luschnathiana & 41 & 4,58 & 8,5 & 4,59 & 4,59 \\
\hline Solanum mauritianum & 21 & 2,35 & 9,5 & 5,14 & 3,74 \\
\hline Casearia silvestris & 22 & 2,46 & 9,0 & 4,86 & 3,66 \\
\hline Cereus hildmannianus & 14 & 1,56 & 5,5 & 2,97 & 2,27 \\
\hline Zanthoxylum rhoifolium & 8 & 0,89 & 4,0 & 2,16 & 1,53 \\
\hline Cecropia pachystachya & 6 & 0,67 & 3,0 & 1,62 & 1,15 \\
\hline Coussapoa microcarpa & 4 & 0,45 & 1,5 & 0,81 & 0,63 \\
\hline Salix humboltiana & 3 & 0,34 & 1,5 & 0,81 & 0,57 \\
\hline Solanum pseudoquina & 3 & 0,34 & 1,5 & 0,81 & 0,57 \\
\hline Patagonula americana & 2 & 0,22 & 1,0 & 0,54 & 0,38 \\
\hline Solanum sanctaecatharinae & 2 & 0,22 & 1,0 & 0,54 & 0,38 \\
\hline Syagrus romanzoffiana & 2 & 0,22 & 1,0 & 0,54 & 0,38 \\
\hline Garcinia gardneriana & 1 & 0,11 & 0,5 & 0,27 & 0,19 \\
\hline Sebastiania serrata & 1 & 0,11 & 0,5 & 0,27 & 0,19 \\
\hline Allophylus edulis & 1 & 0,11 & 0,5 & 0,27 & 0,19 \\
\hline Prunus myrtifolia & 1 & 0,11 & 0,5 & 0,27 & 0,19 \\
\hline Myrtaceae 1 & 1 & 0,11 & 0,5 & 0,27 & 0,19 \\
\hline
\end{tabular}

Trema micrantha, Zanthoxylum rhoifolium e Cereus hildmannianus, com exceção de Casearia silvestris e Ficus luschnathiana, que não germinaram no tratamento com incidência de luz natural na profundidade entre 5 e $10 \mathrm{~cm}$. As nove espécies restantes somaram $6 \%$ do total de indivíduos (tabela 4).

Nos tratamentos na segunda amostragem, $53 \%$ dos indivíduos do BSS 2 germinaram na condição com sombrite (figura 1A). Em relação ao número de espécies, apenas Patagonula americana não germinou nesta condição (tabela 4). Quanto à profundidade, o maior número de indivíduos germinou entre a superfície e $5 \mathrm{~cm}(81 \%)$, assim como o de espécies (16) (figura 1A, B), sendo Solanum pseudoquina restrita a esta profundidade, enquanto que, entre 5 e $10 \mathrm{~cm}$, germinaram 150 indivíduos (19\%) e 11 espécies, com uma espécie exclusiva (Patagonula americana).

O BSS 1 apresentou cinco espécies diferentes em comparação com a outra época (Allophylus edulis, Prunus myrtifolia, Salix humboldtiana, Sebastiania serrata e Syagrus romanzoffiana), enquanto o BSS 2 somente duas (Cupania vernalis e Eugenia rostrifolia). Essas espécies, restritas a cada amostragem, tiveram um baixo número de indivíduos recrutados, não tendo uma representação muito grande no total, visto os valores de importância de cada grupo terem somado $1,52 \%$ e $1,13 \%$, respectivamente (tabelas $2,3)$.

O teste de Tukey mostrou diferenças significativas na média de espécies recrutadas entre os tratamentos do BSS 1, assim como entre as profundidades do tratamento sombra (tabela 5). Nesta amostragem, o maior número de espécies recrutadas foi no tratamento sombra, com maior média, na profundidade de $0-5 \mathrm{~cm}$. No BSS 2, a média de espécies foi superior entre $0-5 \mathrm{~cm}$, decrescendo com o aumento da profundidade (tabela 5). Quanto aos valores do número de sementes por tratamentos e profundidades nas duas amostragens, não houve diferenças significativas (tabela 5).

A estimativa da diversidade de Shannon $\left(H^{\prime}\right)$, para o BSS 1 e BSS 2, resultou em 1,639 (nats indivíduo ${ }^{-1}$ ) e 1,717 (nats indivíduo ${ }^{-1}$ ), e a equabilidade de Pielou $\left(J^{\prime}\right)$ 
Tabela 3. Parâmetros fitossociológicos calculados para as espécies arbóreas recrutadas nas amostras de banco de sementes 2 (BSS 2) em uma floresta estacional no Parque Estadual de Itapuã, Viamão, RS, em ordem decrescente de valor de importância (VI). ( $\mathrm{Ni}=$ número de indivíduos; $\mathrm{DR}=$ densidade relativa; $\mathrm{FA}=$ freqüência absoluta; $\mathrm{FR}=$ freqüência relativa).

Table 3. Phytosociological parameters calculated for the tree species recruited in the samples of soil seed bank 2 (BSS 2) in a seasonal forest at State Park of Itapuã, Municipality of Viamão (RS), ranked according to importance value (VI). (Ni = individuals number; $\mathrm{DR}=$ relative density; $\mathrm{FA}=$ absolute frequency; $\mathrm{FR}=$ relative frequency).

\begin{tabular}{|c|c|c|c|c|c|}
\hline Espécie & $\mathrm{Ni}$ & $\begin{array}{l}\mathrm{DR} \\
(\%)\end{array}$ & $\begin{array}{l}\text { FA } \\
(\%)\end{array}$ & $\begin{array}{l}\mathrm{FR} \\
(\%)\end{array}$ & $\begin{array}{c}\text { VI } \\
(\%)\end{array}$ \\
\hline Banara parviflora & 275 & 34,08 & 50,0 & 25,19 & 29,63 \\
\hline Ficus organensis & 224 & 27,76 & 42,5 & 21,41 & 24,58 \\
\hline Trema micrantha & 162 & 20,07 & 41,5 & 20,91 & 20,49 \\
\hline Casearia silvestris & 54 & 6,69 & 22,5 & 11,34 & 9,01 \\
\hline Zanthoxylum rhoifolium & 17 & 2,11 & 8,5 & 4,28 & 3,19 \\
\hline Cereus hildmannianus & 16 & 1,98 & 7,0 & 3,53 & 2,76 \\
\hline Solanum mauritianum & 12 & 1,49 & 6,0 & 3,02 & 2,26 \\
\hline Cecropia pachystachya & 12 & 1,49 & 5,5 & 2,77 & 2,13 \\
\hline Ficus luschnathiana & 13 & 1,61 & 4,5 & 2,27 & 1,94 \\
\hline Solanum sanctaecatharinae & 6 & 0,74 & 3,0 & 1,51 & 1,13 \\
\hline Coussapoa microcarpa & 6 & 0,74 & 2,5 & 1,26 & 1,00 \\
\hline Cupania vernalis & 4 & 0,50 & 2,0 & 1,01 & 0,75 \\
\hline Garcinia gardneriana & 2 & 0,25 & 1,0 & 0,50 & 0,38 \\
\hline Eugenia rostrifolia & 2 & 0,25 & 1,0 & 0,50 & 0,38 \\
\hline Patagonula americana & 1 & 0,12 & 0,5 & 0,25 & 0,19 \\
\hline Solanum pseudoquina & 1 & 0,12 & 0,5 & 0,25 & 0,19 \\
\hline
\end{tabular}

em 0,557 e 0,574 , respectivamente. Os valores destes dois índices mostraram-se bastante semelhantes nas duas amostragens de solo. O baixo valor da equabilidade deve-se a alta concentração de indivíduos recrutados em um pequeno número de espécies.

$O$ índice de Jaccard revelou a existência de similaridade entre os levantamentos das espécies recrutadas nas duas amostragens do banco de sementes do solo $(0,67)$, com 14 espécies comuns nas duas épocas (setembro/2002 e março/2003). Em relação ao levantamento fitossociológico do componente arbóreo (DAP $\geq 5 \mathrm{~cm}$ ) com as espécies recrutadas no banco de sementes do solo, a similaridade foi baixa $(0,19$ com o BSS 1 e 0,18 com o BSS 2).

Das espécies identificadas que germinaram nas amostras de solo, apenas 12 foram comuns às 44 espécies amostradas no levantamento fitossociológico realizado na área: Allophylus edulis, Banara parviflora, Casearia silvestris, Coussapoa microcarpa, Cupania vernalis, Eugenia rostrifolia, Garcinia gardneriana, Patagonula americana, Prunus myrtifolia, Sebastiania serrata, Solanum sanctaecatharinae e Zanthoxylum rhoifolium (Kray 2004).

\section{Discussão}

As densidades encontradas no presente estudo (entre 74 e 78 sementes $\mathrm{m}^{-2}$ ), dadas pelo número total de sementes viáveis nas amostragens do banco de sementes do solo, situaram-se dentro dos valores relatados para florestas, que variaram de 25 a 3.350 sementes $\mathrm{m}^{-2}$ (Garwood 1989). Comparativamente, trabalhos realizados em floresta ombrófila densa mostraram ter densidades em geral maiores: Baider et al. (1999) encontraram 389 sementes $\mathrm{m}^{-2}$ e Baider et al. (2001) obtiveram 25 , 268, 284 e 389 sementes $\mathrm{m}^{-2}$, em quatro florestas com idades diferentes. Esses dois trabalhos coletaram o solo até $5 \mathrm{~cm}$ de profundidade. Em floresta estacional semidecidual, Grombone-Guaratini \& Rodrigues (2002) encontraram densidades menores que a do presente trabalho (32,3, 49,6 e 46,3 sementes $\left.\mathrm{m}^{-2}\right)$, em estudo que considerou três épocas distintas; com as coletas de solo tendo sido feitas em profundidades de até $3 \mathrm{~cm} \mathrm{e}$ incluindo-se todas as formas de vida na soma dos indivíduos. Em floresta ombrófila mista, Caldato et al. (1996) igualmente encontraram densidades menores em dois tipos florestais $\left(10,4\right.$ e 37,4 sementes $\left.\mathrm{m}^{-2}\right)$, cujas coletas foram realizadas até $3 \mathrm{~cm}$ de profundidade. 
Tabela 4. Distribuição do número de indivíduos recrutados por espécie no banco de sementes do solo (BSS 1; BSS 2), por tipo de tratamento, em uma floresta estacional no Parque Estadual de Itapuã, Viamão, RS.

Table 4. Distribution of the number species recruited from soil seed bank (BSS 1; BSS 2) by treatment type in a seasonal forest at State Park of Itapuã, Municipality of Viamão (RS).

\begin{tabular}{|c|c|c|c|c|c|c|c|c|}
\hline \multirow[t]{4}{*}{ Família/Espécie } & \multicolumn{8}{|c|}{ Tratamento } \\
\hline & \multicolumn{4}{|c|}{ Sombra } & \multicolumn{4}{|c|}{ Luz } \\
\hline & \multicolumn{2}{|c|}{$0-5 \mathrm{~cm}$} & \multicolumn{2}{|c|}{$5-10 \mathrm{~cm}$} & \multicolumn{2}{|c|}{$0-5 \mathrm{~cm}$} & \multicolumn{2}{|c|}{$5-10 \mathrm{~cm}$} \\
\hline & BSS 1 & BSS 2 & BSS 1 & BSS 2 & BSS 1 & BSS 2 & BSS 1 & BSS 2 \\
\hline Ficus organensis & 144 & 94 & 101 & 34 & 53 & 84 & 42 & 12 \\
\hline Banara parviflora & 109 & 115 & 38 & 32 & 4 & 121 & 12 & 7 \\
\hline Trema micrantha & 75 & 53 & 72 & 22 & 48 & 64 & 64 & 23 \\
\hline Casearia silvestris & 15 & 26 & 7 & 5 & 0 & 23 & 0 & 0 \\
\hline Ficus luschnathiana & 22 & 5 & 9 & 1 & 9 & 7 & 1 & 0 \\
\hline Solanum mauritianum & 8 & 6 & 8 & 2 & 5 & 2 & 1 & 2 \\
\hline Cereus hildmannianus & 7 & 7 & 4 & 1 & 1 & 7 & 2 & 1 \\
\hline Zanthoxylum rhoifolium & 5 & 8 & 2 & 2 & 1 & 5 & 0 & 2 \\
\hline Cecropia pachystachya & 4 & 5 & 1 & 1 & 1 & 6 & 0 & 0 \\
\hline Coussapoa microcarpa & 2 & 2 & 2 & 2 & 0 & 2 & 0 & 0 \\
\hline Solanum sanctaecatharinae & 2 & 2 & 0 & 0 & 0 & 3 & 0 & 1 \\
\hline Cupania vernalis** & 0 & 3 & 0 & 0 & 0 & 1 & 0 & 0 \\
\hline Solanum pseudoquina & 0 & 1 & 3 & 0 & 0 & 0 & 0 & 0 \\
\hline Garcinia gardneriana & 1 & 1 & 0 & 0 & 0 & 1 & 0 & 0 \\
\hline Patagonula americana & 2 & 0 & 0 & 0 & 0 & 1 & 0 & 0 \\
\hline Salix humboldtiana* & 0 & 0 & 2 & 0 & 0 & 0 & 1 & 0 \\
\hline Eugenia rostrifolia** & 0 & 1 & 0 & 0 & 0 & 1 & 0 & 0 \\
\hline Syagrus romanzoffiana* & 1 & 0 & 0 & 0 & 1 & 0 & 0 & 0 \\
\hline Allophylus edulis* & 1 & 0 & 0 & 0 & 0 & 0 & 0 & 0 \\
\hline Myrtaceae $1 *$ & 1 & 0 & 0 & 0 & 0 & 0 & 0 & 0 \\
\hline Prunus myrtifolia* & 1 & 0 & 0 & 0 & 0 & 0 & 0 & 0 \\
\hline Sebastiania serrata* & 1 & 0 & 0 & 0 & 0 & 0 & 0 & 0 \\
\hline Totais & 400 & 329 & 249 & 102 & 123 & 328 & 123 & 48 \\
\hline
\end{tabular}

(*) espécies exclusivas do BSS 1 (exclusive species of the BSS 1); (**) espécies exclusivas do BSS 2 (exclusive species of the BSS2).

As diferenças de densidade entre esses trabalhos podem refletir a falta de padronização metodológica, $o$ que limita comparações diretas (Butler \& Chazdon 1998, Dalling et al. 1998, Grombone-Guaratini \& Rodrigues 2002), mas também podem resultar de variações na estrutura das florestas, da entrada de diásporos de diferentes locais pela ação de dispersores e da longevidade das sementes no solo (Dalling et al. 1997, 1998, Grombone-Guaratini \& Rodrigues 2002).

Neste estudo, nas duas épocas de coleta, não houve grandes diferenças nos números de espécies e de indivíduos recrutados, assim como na densidade de sementes. Isso provavelmente ocorreu por que as espécies que apresentaram os maiores valores de indivíduos recrutados, nas duas amostragens, possuem sementes de tamanho pequeno, facilmente incorporáveis ao solo. Essas sementes podem permanecer latentes por períodos prolongados no solo, vindo a germinar assim que encontrarem condições propícias, constituindo um componente importante no banco permanente de sementes (Foster \& Janson 1985, Roizman 1993). A estacionalidade climática é identificada como uma das pressões seletivas que determinam a frutificação sazonal das plantas em comunidades (Morellato 1995). A pouco pronunciada sazonalidade climática na área pode definir um padrão contínuo de frutificação (Morellato et al. 2000), refletido na semelhança do banco entre estações. Grombone-Guaratini \& Rodrigues (2002) citam a ocorrência de valores de densidade sem diferenças significativas nas duas amostragens do banco de sementes do solo realizadas no mesmo ano, mas diferem de uma amostragem feita no ano anterior, provavelmente 


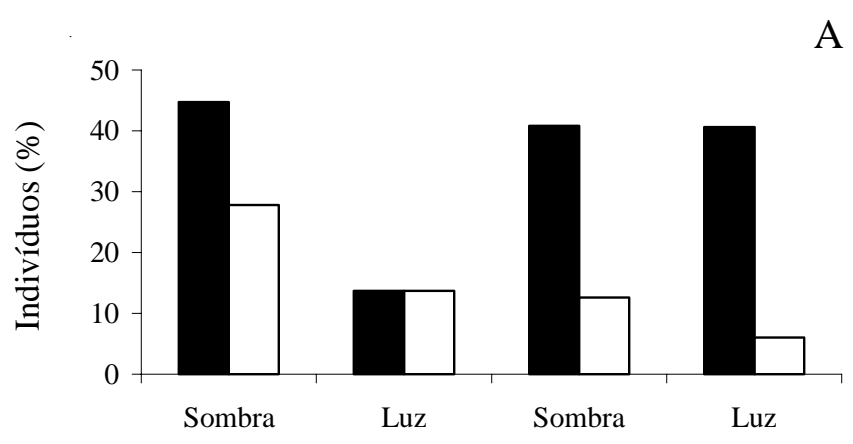

B

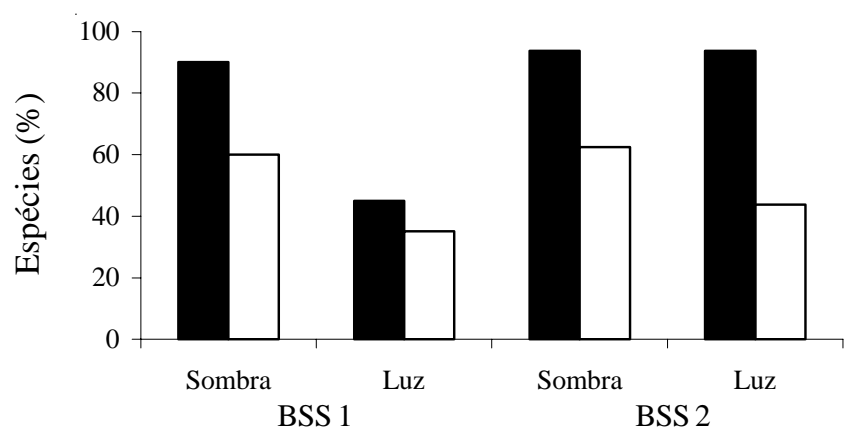

Figura 1. Porcentagem de indivíduos (A) e de espécies (B) recrutadas por profundidades $(\boldsymbol{\square}=0 \mathrm{a} 5 \mathrm{~cm} ; \square=5 \mathrm{a} 10 \mathrm{~cm}) \mathrm{e}$ tratamentos nas amostras do banco de sementes do solo (BSS 1; BSS 2) em uma floresta estacional no Parque Estadual de Itapuã, Viamão, RS.

Figure 1. Percentage of recruited individuals (A) and species (B) accord to soil depth $(\boldsymbol{\square}=0$ at $5 \mathrm{~cm} ; \square=5$ at $10 \mathrm{~cm})$ and treatments, from samples of soil seed bank (BSS 1; BSS 2) in a seasonal forest at State Park of Itapuã, municipality of Viamão (RS).
A devido à variação na chuva de sementes provocada por diferenças nas condições meteorológicas entre anos. Flutuações na produção de sementes por uma comunidade, sazonal ou anualmente, podem influenciar a composição e abundância de sementes no solo (Putz \& Appanah 1987, Dalling et al. 1997, Butler \& Chazdon 1998).

A amostragem do banco de sementes do solo não apresentou similaridade com o levantamento fitossociológico do componente arbóreo realizado na área por Kray (2004), tendo sido encontradas diversas espécies que estiveram ausentes no banco, assim como aquelas intolerantes à sombra (pioneiras) que estão no solo, mas não foram amostradas na área. Tanto em florestas temperadas como em tropicais, raramente encontra-se similaridade florística entre o estoque de sementes e a vegetação local (Martínez-Ramos \& SotoCastro 1993, Roizman 1993). A dissimilaridade apresentada pode ser devida, para algumas espécies, à formação de um banco de plântulas ao invés do de sementes no solo (Garwood 1989, George \& Bazzaz 1999, Antos et al. 2005), pela predação de sementes maiores ou por dificuldade destas incorporarem-se ao solo (Putz \& Appanah 1987). As espécies em amostras de solo que germinam, geralmente estão ausentes ou são raras na vegetação local, e provêm de diferentes locais e épocas (Garwood 1989), justificando a importância da presença de dispersores para o transporte de propágulos na recomposição florestal. A dispersão de sementes sobre uma área degradada é essencial para a sua regeneração, uma vez que o banco de sementes do solo sofre uma rápida diminuição na abundância e riqueza de espécies devido à curta viabilidade de muitas delas (Garwood 1989).

Tabela 5. Comparações entre médias realizadas aos pares no banco de sementes do solo (BSS 1; BSS 2), entre as profundidades $(0-5 \mathrm{~cm} ; 5-10 \mathrm{~cm})$ e entre os tratamentos (luz; sombra), em uma floresta estacional no Parque Estadual de Itapuã, Viamão, RS. Valores seguidos por letras diferentes indicam variações significativas $(P<0,05)$. ns = não significativo

Table 5. Comparisons between paired means obtained from the soil seed bank (BSS 1; BSS 2), depths (0-5 cm; 5-10 cm) and treatments (light; shade) in a seasonal forest at State Park of Itapuã, municipality of Viamão (RS). Values followed by different letters indicate significant variations $(P<0.05) . \mathrm{ns}=$ not significant

\begin{tabular}{|c|c|c|c|c|}
\hline & $\operatorname{Luz}(0-5 \mathrm{~cm})$ & Sombra $(0-5 \mathrm{~cm})$ & $\operatorname{Luz}(5-10 \mathrm{~cm})$ & Sombra $(5-10 \mathrm{~cm})$ \\
\hline \multicolumn{5}{|l|}{ BSS 1} \\
\hline No de espécies & $1,06 \pm 0,9 \mathrm{a}$ & $3,2 \pm 1,3 \mathrm{~b}$ & $1,1 \pm 0,8 \mathrm{a}$ & $2,06 \pm 1,2 \mathrm{c}$ \\
\hline $\mathrm{N}^{\mathrm{o}}$ de sementes & $13,6 \pm 21 \mathrm{~ns}$ & $22,2 \pm 42,1 \mathrm{~ns}$ & $17,5 \pm 25,3 \mathrm{~ns}$ & $20,75 \pm 32,8 \mathrm{~ns}$ \\
\hline \multicolumn{5}{|l|}{ BSS 2} \\
\hline $\mathrm{N}^{\mathrm{o}}$ de espécies & $3 \pm 1,4 \mathrm{a}$ & $3 \pm 1,1 \mathrm{a}$ & $0,7 \pm 0,9 \mathrm{~b}$ & $1,1 \pm 0,9 \mathrm{~b}$ \\
\hline $\mathrm{N}^{\mathrm{o}}$ de sementes & $21,8 \pm 37,1 \mathrm{~ns}$ & $21,9 \pm 36,4 \mathrm{~ns}$ & $6,8 \pm 8,1 \mathrm{~ns}$ & $10,2 \pm 13,5 \mathrm{~ns}$ \\
\hline
\end{tabular}


Poucos estudos usam em suas análises mais de um tratamento, prevalecendo os que trabalham sob condição de luz natural. Os resultados obtidos neste trabalho assemelham-se aos de Caldato et al. (1996), que também usaram dois tratamentos e obtiveram maior número de indivíduos germinados na condição de sombreamento (sombrite $70 \%$ ).

A ocorrência de um maior número de espécies e indivíduos na condição com sombrite no presente estudo, principalmente no BSS 1, pode ser consequiência das altas temperaturas no interior da casa de vegetação, visto que este experimento ocorreu durante o final da primavera e todo o verão (outubro a março). A germinação, em especial nas amostras de solo submetidas ao tratamento com incidência de luz natural, pode ter sido prejudicada por este fator, enquanto na condição de sombreamento, as temperaturas teriam sido mais amenas. Porém, esta hipótese não pode ser confirmada, por não terem sido realizadas medidas de temperatura. Além disso, estudos referentes à germinação de espécies nativas são escassos, o que torna difícil justificar fatores e intensidades que estimulem ou inibam a germinação de sementes.

Quanto à distribuição vertical das sementes no banco, Roizman (1993) também encontrou o maior número de indivíduos e espécies entre a superfície e $5 \mathrm{~cm}$ de profundidade e, nas demais profundidades $(5 \mathrm{a}$ $10 \mathrm{~cm}$ e 10 a $15 \mathrm{~cm}$ ) houve um decréscimo tanto no número de espécies quanto no de indivíduos. A distribuição vertical do banco de sementes se dá por ação de diferentes mecanismos bióticos e abióticos de incorporação no solo, cujas origens são variadas e, na maioria das vezes, pouco conhecidas, apesar de serem fundamentais para a compreensão da heterogeneidade espacial do banco e da latência das sementes presentes (Garwood 1989). Constatou-se que a metodologia usada diferiu entre trabalhos, como a amostragem na profundidade entre a superfície e $3 \mathrm{~cm}$ (Caldato et al. 1996, Grombone-Guaratini \& Rodrigues 2002) ou por realizarem duas coletas em profundidades diferentes (superfície a 2,5 cm e 2,5 a $5 \mathrm{~cm}$ ) (Baider et al. 1999, 2001). O fato de encontrarem-se sementes viáveis nas camadas mais profundas do solo indica a existência de mecanismos que promovem a longevidade de algumas espécies nesta condição. Adormência proporciona maior longevidade e permite que sementes de algumas espécies permaneçam latentes e sobrevivam sob condições adversas para, posteriormente, germinarem e estabelecerem-se (Popinigis 1985).

Das três espécies que apresentaram os maiores números de indivíduos germinados nas duas amostragens de solo, apenas Banara parviflora foi registrada no levantamento fitossociológico da área (Kray 2004). A presença das outras duas, Ficus organensis e Trema micrantha, pode ser pela eficiência de seus mecanismos de dispersão, pela ocorrência de árvores nas proximidades da área ou ainda, podem ter ocorrido anteriormente no local, tendo as sementes permanecido dormentes no banco de sementes do solo. A presença de Ficus organensis também pode ser justificada pela ocorrência de um ou mais indivíduos hemi-epífitos, não registrados no levantamento fitossociológico do componente arbóreo realizado por Kray (2004). Estas espécies possuem sementes pequenas, apresentando vantagens adaptativas por sofrerem menor pressão de predação e terem maior facilidade de incorporação ao estoque do solo.

A presença de Cecropia pachystachya, Solanum mauritianum e Trema micrantha na composição do banco de sementes do solo, cujos gêneros são citados como característicos de fases iniciais do processo de sucessão (Baider et al. 1999, 2001, Grombone-Guaratini \& Rodrigues 2002), revelam o potencial de regeneração da floresta, em caso de abertura de clareiras ou outro fator desestabilizador que venha alterar a estrutura presente. Rodrigues (1995) salienta que espécies pioneiras atuam como cicatrizadoras de ambientes perturbados e apresentam como característica ecológica a grande eficiência na distribuição de suas sementes por toda a floresta, podendo ficar dormentes no solo (banco de sementes) ou serem continuamente dispersas pelos animais entre clareiras de diferentes idades.

Espécies características de etapas mais avançadas do processo de regeneração, como Banara parviflora, Casearia silvestris, Coussapoa microcarpa, Ficus organensis e F. luschnathiana (Baider et al. 1999, 2001, Gandolfi 2000), igualmente constatadas na amostragem deste trabalho, indicam, por outro lado, que em caso de um "distúrbio" local, estas potencialmente poderão tomar parte no processo de recomposição, a medida que condições forem criadas para o seu desenvolvimento.

A diversidade específica obtida para as amostragens do banco de sementes $\left(H^{\prime}=1,639-\mathrm{BSS} 1\right.$ e $H^{\prime}=1,717$ - BSS 2) reflete o baixo número de espécies e a baixa equabilidade, esta motivada pela grande concentração de indivíduos germinados em um pequeno número de espécies. Grombone-Guaratini \& Rodrigues (2002), em uma das estações de coleta (estação seca/1996), encontraram valores mais altos $\left(H^{\prime}=2,24\right.$ e $\left.J^{\prime}=0,59\right)$, proporcionados por maior riqueza e equabilidade entre as espécies, possivelmente como conseqüência da 
inclusão de outras formas de vida, além de árvores. Caldato et al. (1996) em dois tipos florestais (com e sem predominância da araucária), da mesma forma, encontraram valores baixos $\left(H^{\prime}=1,86\right.$ e $J^{\prime}=0,68$, $H^{\prime}=1,68$ e $J^{\prime}=0,58$, respectivamente). A diversidade $\left(H^{\prime}\right)$ do banco de sementes mostra-se baixa quando comparadas às estimadas em levantamentos fitossociológicos do componente arbóreo em florestas na região, que variam de 1,886 a 3,548 (nats indivíduo-1 ${ }^{-1}$ (Vasconcellos et al. 1992, Bencke \& Soares 1998, Waechter \& Jarenkow 1998, Waechter et al. 2000, Jarenkow \& Waechter 2001, Jurinitz \& Jarenkow 2003). Para o levantamento fitossociológico realizado na área a diversidade foi de 2,665 (nats indivíduo-1) e a equabilidade de 0,708 (Kray 2004).

Estudos que analisem os diferentes tipos de perturbações, interações com os animais, bem como, informações detalhadas sobre a fenologia e a ecofisiologia da germinação de espécies arbóreas são necessários para o entendimento dos processos de restauração de comunidades florestais e definição de estratégias de conservação. Atualmente há uma carência de trabalhos publicados sobre a regeneração natural em florestas sul-brasileiras, portanto, estudos semelhantes devem ser incentivados, com padronização e adequação da metodologia para permitir análises comparativas.

Agradecimentos - Ao programa de Pós-Graduação em Botânica da UFRGS, pela possibilidade de realização deste trabalho; ao CNPq (Processo 472960/03-2), pela bolsa concedida à primeira autora; à Fundação $\mathrm{O}$ Boticário de Proteção à Natureza, pelo apoio ao projeto; ao DUC/DEFAP/SEMA-RS, pelo apoio logístico nas dependências do Parque; ao Departamento de Solos da Faculdade de Agronomia/UFRGS, pelo empréstimo das instalações na casa de vegetação; aos Biólogos M. Sobral, pela determinação de algumas espécies, e Jean C. Budke, pela ajuda na análise estatística dos dados; ao Prof. J.L. Waechter, por valiosas sugestões.

\section{Referências bibliográficas}

ANTOS, J.A., GUEST, H.J. \& PARISH, R. 2005. The tree seedling bank in an ancient montane forest: stress tolerators in a productive habitat. Journal of Ecology 93: 536-543.

ALVAREZ-BUYLLA, E.R. \& MARTÍNEZ-RAMOS, M. 1990. Seed bank versus seed rain in the regeneration of a tropical pioneer tree. Oecologia 84:314-325.

APG II (ANGIOSPERM PHYLOGENY GROUP). 2003. An update of the Angiosperm Phylogeny Group classification for orders and families of flowering plants: APG II. Botanical Journal of the Linnean Society 141:399-436.
BAIDER, C., TABARELLI, M. \& MANTOVANI, W. 1999. O banco de sementes de um trecho de Floresta Atlântica Montana, São Paulo, Brasil. Revista Brasileira de Biologia 59:319-328.

BAIDER, C., TABARELLI, M. \& MANTOVANI, W. 2001. The soil seed bank during Atlantic Forest regeneration in southeast Brazil. Revista Brasileira de Biologia 61:35-44.

BENCKE, C.S.C. \& SOARES, C. 1998. Estudo fitossociológico da vegetação arbórea de uma área de floresta estacional em Santa Cruz do Sul, RS, Brasil. Caderno de Pesquisa, Série Botânica 10:37-57.

BROWN, D. 1992. Estimating the composition of a forest seed bank: a comparison of the seed extraction and seedling emergence methods. Canadian Journal of Botany 70:1603-1612.

BUTLER, B.J. \& CHAZDON, R.L. 1998. Species richness, spatial variation, and abundance of soil seed bank of a secondary tropical rain forest. Biotropica 30:214-222.

CALDATO, S.L., FLOSS, P.A., CROCE, D.M. \& LONGHI, S.J. 1996. Estudos da regeneração natural, banco de semente e chuva de sementes na Reserva Genética Florestal de Caçador, SC. Ciência Florestal 6:27-38.

CALLEGARI-JACQUES, S.M. 2004. Bioestatística: princípios e aplicações. Artmed, Porto Alegre.

DALLING, J.W., SWAINE, M.D. \& GARWOOD, N.C. 1997. Soil seed bank community dynamics in seasonally moist lowland tropical forest, Panama. Journal of Tropical Ecology 13:659-680.

DALLING, J.W., SWAINE, M.D. \& GARWOOD, N.C. 1998. Dispersal patterns and seed bank dynamics of pioneer trees in moist tropical forest. Ecology 79:564-578.

DANIEL, O. \& JANKAUSKIS, J. 1989. Avaliação de metodologia para o estudo do estoque de sementes do solo, em floresta de terra firme na Amazônia brasileira. IPEF 41/42:18-26.

ESPÍNDOLA, M.B., VIEIRA, N.K. \& REIS, A. 2003. A chuva e o banco de sementes na restauração de ecossistemas. In Anais do VI Congresso de Ecologia do Brasil, Fortaleza, p.562-564.

FENNER, M. 1985. Soil seed banks. In Seed ecology. (M. Fenner, ed.). Chapman \& Hall, London. p.57-71.

FOSTER, S.A. \& JANSON, C.H. 1985. The relationship between seed size and establishment conditions in tropical woody plants. Ecology 66: 773-780.

GANDOLFI, S. 2000. História natural de uma floresta estacional semidecidual no município de Campinas (São Paulo, Brasil). Tese de doutorado, Universidade Estadual de Campinas, Campinas.

GARWOOD, N.C. 1989. Tropical soil seed banks: a review. In Ecology of soil seed banks (M.A. Leck, V.T. Parker \& R.L. Simpson, eds.). Academic Press, San Diego, p.149-209.

GEORGE, L.O. \& BAZZAZ, F.A. 1999. The fern understory as an ecological filter: growth and the survival of canopytree seedlings. Ecology 80:846-856. 
GRAHAM, A.W. \& HOPKINS, M.S. 1990. Soil seed banks of adjacent unlogged rainforest types in north Queesnsland. Australian Journal of Botany 38:261-268.

GROMBONE-GUARATINI, M.T. \& RODRIGUES, R.R. 2002. Seed bank and seed rain in a seasonal semi-deciduous forest in south-eastern Brazil. Journal of Tropical Ecology 18:759-774.

GROSS, K.L.A. 1990. A comparison of methods for estimating seed numbers in the soil. Journal of Ecology 78:1079-1093.

HALL, J.B. \& SWAINE, M.D. 1980. Seed stocks in Ghanaian forest soils. Biotropica 12:256-263.

HARPER, J.L. 1977. Population biology of plants. Academic Press, London.

JARENKOW, J.A. \& WAECHTER, J.L. 2001. Estrutura de floresta estacional no Rio Grande do Sul. Revista Brasileira de Botânica 24:263-272.

JURINITZ, C.F. \& JARENKOW, J.A. 2003. Estrutura do componente arbóreo de uma floresta estacional na Serra do Sudeste, Camaquã (RS), Brasil. Revista Brasileira de Botânica 26:475-487.

KRAY, J.G. 2004. Estrutura e diversidade arbórea da floresta estacional no Parque Estadual de Itapuã, Viamão, RS, Brasil. Monografia de Bacharelado, Universidade Federal do Rio Grande do Sul, Porto Alegre.

MAGURRAN, A.E. 1988. Ecological diversity and its measurement. Princeton University Press, Princeton.

MARTÍNEZ-RAMOS, M. \& SOTO-CASTRO, A. 1993. Seed rain and advanced regeneration in a tropical rain forest. Vegetatio 107/108:299-318.

MORELLATO, L.P.C. 1995. As estações do ano na floresta. In Ecologia e preservação de uma floresta tropical urbana: Reserva de Santa Genebra (H.F. Leitão Filho \& L.P. Morellato, eds.). Editora da Unicamp, Campinas, p.37-41.

MORELLATO, L.P.C., TALORA, D.C., TAKAHASI, A., BENCKE,C.C. \& ROMERA, E.C. 2000. Phenology of atlantic rain forest trees: a comparative study. Biotropica 32:811-823.

MORENO, J.A. 1961. Clima do Rio Grande do Sul. Secretaria da Agricultura do Rio Grande do Sul, Porto Alegre.

MUELLER-DOMBOIS, D. \& ELLENBERG, H. 1974. Aims and methods of vegetation ecology. John Willey and Sons, New York.

POPINIGIS, F. 1985. Fisiologia da semente. $2^{\text {a }}$ ed. Agiplan, Brasília.
PUTZ, F.E. \& APPANAH, S. 1987. Buried seeds, newly dispersed seed, and the dynamics of a lowland forest in Malasia. Biotropica 19:326-333.

RIO GRANDE DO SUL. 1997. Plano de manejo do Parque Estadual de Itapuã, RS. Departamento de Recursos Renováveis/Pró-Guaíba, Porto Alegre.

RODRIGUES, R.R. 1995. A sucessão florestal. In Ecologia e preservação de uma floresta tropical urbana: Reserva de Santa Genebra. (H.F. Leitão Filho \& L.P. Morellato, eds.). Editora da Unicamp, Campinas, p.30-35.

ROIZMAN, L.G. 1993. Fitossociologia e dinâmica do banco de semente de populações arbóreas de florestas secundárias em São Paulo, SP. Dissertação de mestrado, Universidade de São Paulo, São Paulo.

SOLIANI JR., E., KOESTER, E. \& FERNÁNDEZ, L.A. 2000. A geologia isotópica do escudo sul-riograndense. In Geologia do Rio Grande do Sul (M. Holz \& L.F. Ros, eds.). CIGO/UFRGS, Porto Alegre, p.175-230.

THOMPSON, K. \& GRIME, J.P. 1979. Seasonal variation in the seed banks of herbaceous species in ten contrasting habitats. Journal of Ecology 67:893-921.

VASCONCELLOS, J.M.O., DIAS, L.L., SILVA, C.P.\& SOBRAL, M. 1992. Fitossociologia de uma mata subtropical no Parque Estadual do Turvo, RS. Revista do Instituto Florestal 4:252-259.

VELOSO, H.P. \& GÓES-FILHO, L. 1982. Fitogeografia brasileira: classificação fisionômica-ecológica da vegetação neotropical. Boletim Técnico Projeto RADAMBRASIL, Série. Vegetação, 1:1-80.

WAECHTER, J.L. \& JARENKOW, J.A. 1998. Composição e estrutura do componente arbóreo nas matas turfosas do Taim, Rio Grande do Sul. Biotemas 11:45-69.

WAECHTER, J.L., MÜLLER, S.C., BREIER, T.B. \& VENTURI, S. 2000. Estrutura do componente arbóreo em uma floresta subtropical de planície costeira interna. In Anais do V Simpósio de Ecossistemas Brasileiros: Conservação (S. Watanabe, coord.). Aciesp, São Paulo. v. 3, p.92-112.

WILLIAMS-LINERA, G. 1993. Soil seed banks in four lower montane forests of Mexico. Journal of Tropical Ecology 9:321-337.

YOUNG, K.R., EWEL, J.J. \& BROWN, B.J. 1987. Seed dynamics during forest succession in Costa Rica. Vegetatio 71:157-173.

ZAR, J. 1999. Biostatistical analysis. $4^{\text {th }}$ ed. Prentice-Hall, New Jersey. 